\title{
Dietary Intake at 9 Years and Subsequent Body Mass Index in Adolescent Boys and Girls: A Study of Monozygotic Twin Pairs
}

\author{
Lise Dubois, ${ }^{1}$ Maikol Diasparra, ${ }^{1}$ Leonie-Helen Bogl, ${ }^{2}$ Bénédicte Fontaine-Bisson, ${ }^{3}$ Brigitte Bédard, ${ }^{1}$ \\ Richard E. Tremblay, ${ }^{4,5,6,7}$ Jaakko Kaprio, ${ }^{2,8,9}$ and Michel Boivin ${ }^{10,7}$ \\ ${ }^{1}$ School of Epidemiology, Public Health and Preventive Medicine, University of Ottawa, Ottawa, ON, Canada \\ ${ }^{2}$ Department of Public Health, University of Helsinki, Helsinki, Finland \\ ${ }^{3}$ School of Nutrition Sciences, University of Ottawa, Ottawa, ON, Canada \\ ${ }^{4}$ Research Unit on Children's Psychosocial Maladjustment, Sainte-Justine Hospital Research Centre, Montreal, OC, Canada \\ ${ }^{5}$ Department of Pediatrics and Psychology, University of Montreal, Montreal, OC, Canada \\ ${ }^{6}$ School of Public Health, Physiotherapy and Population Science, University College Dublin, Dublin, Ireland \\ ${ }^{7}$ Institute of Genetic, Neurobiological and Social Foundations of Child Development, Tomsk State University, Tomsk, \\ Russian Federation \\ ${ }^{8}$ Department of Health, National Institute for Health and Welfare, Helsinki, Finland \\ ${ }^{9}$ Institute for Molecular Medicine (FIMM), University of Helsinki, Helsinki, Finland \\ ${ }^{10}$ School of Psychology, Université Laval, Québec, QC, Canada
}

\begin{abstract}
There is a lack of evidence pointing to specific dietary elements related to weight gain and obesity prevention in childhood and adulthood. Dietary intake and obesity are both inherited and culturally transmitted, but most prospective studies on the association between diet and weight status do not take genetics into consideration. The objective of this study was to document the association between dietary intake at 9 years and subsequent Body Mass Index (BMI) in adolescent monozygotic boy and girl twin pairs. This research used data from 152 twin pairs. Dietary data were collected from two 24-hour-recall interviews with a parent and the child aged 9 years. Height and weight were obtained when the twins were aged $9,12,13$, and 14 years. Intrapair variability analysis was performed to identify dietary elements related to BMI changes in subsequent years. BMI-discordant monozygotic twin pairs were also identified to analyze the dietary constituents that may have generated the discordance. After eliminating potential confounding genetic factors, pre-adolescent boys who ate fewer grain products and fruit and consumed more high-fat meat and milk had higher BMls during adolescence; pre-adolescent girls who consumed more grain products and high-fat meat and milk had higher BMls during adolescence. Energy intake (EI) at 9 years was not related to BMI in subsequent years. Our study suggests that messages and interventions directed at obesity prevention could take advantage of sex-specific designs and, eventually, genetic information.
\end{abstract}

Keywords: twins, monozygotic, diet, BMI, adolescents, longitudinal

Overweight and obesity are important risk factors for chronic diseases and premature death (Crino et al., 2015; Guh et al., 2009; Hagg et al., 2015; Speiser et al., 2005). Given that childhood overweight and obesity are associated with obesity in adulthood (Garver et al., 2013; Monteiro \& Victora, 2005), obesity prevention should begin as early in life as possible. The incentives embedded in the food supply, which promotes over-nutrition, may be one of the main drivers of the obesity epidemic (Swinburn et al., 2011). In this context, dietary intake is an environmental factor that still needs to be better understood in relation to obesity prevalence at the individual and population levels (Sorensen, 2009).
In recent years, researchers have concentrated on identifying the dietary elements that promote excessive weight gain in populations. The results of these studies, however, remain inconclusive: specifically, recent systematic reviews reported a lack of evidence pointing to specific dietary elements (as well as physical activity elements) that were

RECEIVED 13 November 2015; ACCEPTED 26 November 2015.

ADDRESS FOR CORRESPONDENCE: Professor L. Dubois, School of Epidemiology, Public Health and Preventive Medicine, University of Ottawa, 600 Peter Morand Crescent (312C), Ottawa, ON, Canada K1G 5Z3. E-mail: ldubois@uottawa.ca 
related to weight gain and obesity prevention in childhood and adulthood (ESPGHAN Committee on Nutrition et al., 2011; Fogelholm et al., 2012; Summerbell et al., 2009). This lack of evidence was due in part to inconsistencies in research results - at times positive, at others negative or null — arising from differences in survey designs, in methods of dietary intake or body weight assessments, and/or in covariates used in multivariate analyses. Moreover, the majority of these studies presented sex-adjusted data that masked sex and gender specificity (Verdonk \& Klinge, 2012). But more important, even if dietary intake and obesity are both inherited and culturally transmitted (Dubois et al., 2013; Garver et al., 2013; Pallister et al., 2014), the analyses generally did not take genetics into consideration (Bogl et al., 2009; Naukkarinen et al., 2012; Summerbell et al., 2009), which is probably one major reason that a knowledge gap exists today.

Our study was based on different premises. Although both dietary intake and BMI are culturally driven and partly influenced by genetics, for the time being it is simpler to modify eating behaviors than genetics. Adolescence is a period of rapid growth, critical for the development of obesity (Dietz, 1994). Around puberty, sex-specific dietary and body weight characteristics have not been thoroughly studied (Burt Solorzano \& McCartney, 2010). Finally, genetic factors could act as a confounder in the association between dietary intake and BMI. With these premises in mind, the objective of this study was to document the association between dietary intake at 9 years and subsequent BMI in adolescent monozygotic (MZ) boy and girl twin pairs, independent of genetic factors (i.e., DNA sequence), age, sex, and shared environmental factors (e.g., fetal life, family type, socio-economic status, school environment).

\section{Materials and Methods}

\section{Studied Population and Data Collection}

This research used data from the Quebec Newborn Twin Study (QNTS), an ongoing prospective population-based birth cohort of 662 pairs of twins, 50\% boys and 50\% girls, born in Quebec from 1996 to 1998 (Boivin et al., 2013). Dietary data were collected as part of a nutrition study performed when the twins were aged 9 years (mean age 8.96, $S D$ $=0.56$ ). The nutrition survey method has been described elsewhere (Dubois et al., 2013). In short, registered dietitians performed face-to-face 24 -hour-recall interviews with a parent and the child. To counter potential zygosity bias, different interviewers saw Twin 1 and Twin 2 from each pair at 2-week intervals, but on the same days of the week to ensure identical dietary intake reference periods. Twins were assigned randomly to the first interview. The nutrition data for each twin was then captured using Évaluation nutritionnelle, version 1.1.84 (Micro Gesta Inc., QC, Canada), in order to assess the quantity of energy, macronutrients, and specific food items consumed each day, based on the Canadian food composition database, Canadian Nutrient File, version 2005 (Health Canada, 2005).

Data on height and weight were obtained from interviews conducted when the twins were aged $9,12,13$, and 14 years. At age 9 , height and weight were measured at the time of the nutrition survey. At 12,13, and 14 years, height and weight were self-reported. On all occasions, data collection and coding were done randomly and blindly to ensure that neither Twin 1 nor Twin 2 could be identified. Parents and children gave their informed consent before participating in any part of the study. Approval by the Ethics Committee of the Sainte-Justine Hospital Research Centre was obtained before each data collection. Approval for the nutrition study was also obtained from the University of Ottawa Ethics Committee.

We assessed misreporting of dietary intakes by comparing the mean EI derived from dietary recalls and the basal metabolic rate (BMR) estimated using Schofield's equation (Zemel et al., 1997). Based on Goldberg's cut-off equation, under-reporters and over-reporters were defined as having EI:BMR ratios of $<0.96$ and $>2.49$, respectively (Black, 2000). A total of 17 misreporters (9 under-reporters and 8 over-reporters) were identified and then excluded from the analysis. For self-reported anthropometric measurements, we first removed all implausible values (e.g., lower height at 12 years than at 9 years), resulting in the exclusion of a total of 25 twins (17 pairs) at various ages. We then corrected selfreported height and weight data for bias by adjusting them to accord with measured data parameters (Connor Gorber et al., 2009). Finally, we calculated BMI values $\left(\mathrm{kg} / \mathrm{m}^{2}\right)$ for all ages and then calculated changes in BMI for ages 9 through 14 years. Overweight/obesity status was determined based on age- and sex-specific cut-offs for BMI (Cole et al., 2000).

Mean daily intakes of energy (kcal), macronutrients (in $\mathrm{g}$ and as percentage of energy), and various types of food (in kcal and as \% of energy) were estimated. A total of seven main food groups were derived in accordance with Canadian food-based recommendations and nutritional criteria. Subgroups of particular interest were also included in the analysis. Overall, our food groups and subgroups represent $93 \%$ of daily EI. We did not include the remaining $7 \%$ in the analysis because doing so would have yielded a long list of miscellaneous dietary items that would not have been germane to the study.

\section{Statistical Analysis}

Only twins for whom we had dietary data, height and weight measurements at baseline ( 9 years), and subsequent height and weight values (at 12,13, and/or 14 years) were part of the analysis. Since we observed significant interactions for sex and various food groups, we will present results for all $\mathrm{MZ}$ twins and twins by sex. Analyses were adjusted for age (in days) in order to control for time intervals between data 
collections for Twin 1 and Twin 2 (typically 2 weeks apart). A total of $138 \mathrm{MZ}$ twin boys and $166 \mathrm{MZ}$ twin girls were included in the baseline analysis (i.e., dietary intake at 9 years).

Mean daily dietary intake at age 9, BMI at ages 9, 12, 13, and 14 , and changes in BMI at these ages were computed and compared by sex using Wald's $F$ test (when the original variable [or its rank or power transformation] was normally distributed) or a Kruskal-Wallis test (when not normally distributed).

MZ twins constitute an important feature of twin studies because their genetic sequences are identical. In childhood studies, MZ twins living together also match each other in terms of age, sex, and shared environmental factors (e.g., fetal life, family type, socio-economic status, school environment). Each twin then serves as a perfect control for a host of features occurring in his/her co-twin. Differences between twins may thus be ascribed to non-genetic or nonshared environmental influences. If, for instance, intrapair diet differences are associated with BMI differences, the association is more likely to be causal, given that it cannot be accounted for by confounding genetic and familial factors.

For this study, we took advantage of the similarities between MZ twins to perform two types of analyses. First, we compared Twin 1 and Twin 2 in each pair (intrapair variability analysis) using Spearman's correlation, to identify dietary elements related to BMI changes in subsequent years. We used Spearman's coefficient correlation because, unlike Pearson's correlation, Spearman's correlation is less sensitive to univariate (marginal) outliers. More specifically, we looked at the correlations between intrapair differences in dietary intakes at 9 years (Twins 2 minus Twins 1) and intrapair differences in subsequent BMIs ( $\Delta=$ Twins $1 \mathrm{mi}-$ nus Twins 2). Twin 1 and Twin 2 were randomly assigned for this analysis.

Second, we assessed whether or not intrapair differences in dietary intake in BMI-discordant twin pairs were due to chance at each age. For this analysis, the intrapair differences were calculated by subtracting the leaner co-twin's value from the heavier co-twin's value ( $\Delta=$ heavier twin minus leaner twin). MZ BMI-discordant twin pairs were defined as MZ twin pairs differing by at least 2 BMI units. Next, we performed the same type of analysis for concordant twin pairs. Finally, we compared discordant and concordant twin pairs. In all cases, intrapair comparisons of leaner and heavier twins were based on pairs matched using a Wilcoxon test, while between-pair comparisons of discordant and concordant twins were based on a Mann-Whitney U test.

After applying our criterion (a difference of 2 BMI units or more) at $9,12,13$, and 14 years, we identified $4,12,12$, and 6 pairs of discordant MZ girls, and 9, 12, 13, and 12 pairs of discordant MZ boys, respectively. For the same ages, we identified $78,56,53$, and 54 pairs of concordant girls, and $60,39,35$, and 34 pairs of concordant boys, respectively.
We also derived a variable for distinguishing twin pairs that were discordant at least once at these ages, but included only pairs where the heavier twin was consistently heavier than his/her co-twin (if discordant more than once). With this method, we identified 21 pairs of girls and 27 pairs of boys who were discordant at least once at 9, 12, 13, or 14 years, and 62 pairs of girls and 42 pairs of boys who were always concordant at these ages.

All statistical analyses were performed using SAS 9.3 (SAS Institute Inc., Cary, NC, USA). The level of significance was set at 0.05 . Because our sample is small, we will also present results for $0.05 \leq p<.10$ for correlations and MZ discordant pair analyses.

\section{Results}

\section{Descriptive Analysis of $M Z$ Twins}

Table 1 describes daily dietary intake in 9-year-old twins and differences in BMI between ages for all MZ twins and by sex. Compared to boys, girls consumed less total energy as a result of lower macronutrient intake $(\mathrm{g})$ and lower consumption of various food groups (kcal). Although girls had lower dietary intakes overall, food choices appeared similar across sexes.

Compared to boys, girls had lower BMIs at 9 years but higher BMIs at 13 years. Girls also had greater increases in BMIs from 12 to 13 years and from 9 to 14 years. Overall, the prevalence of overweight/obesity in our sample of $\mathrm{MZ}$ twins was $16.1 \%$ at 9 years and varied from $19.6 \%$ to $20.8 \%$ from 12 to 14 years (data not shown).

\section{Intrapair Variability in MZ Twin Pairs}

Tables 2 and 3 compare Twin 1 and Twin 2 in MZ twin pairs. These tables analyze the reciprocal relation between dietary intake and BMI using Spearman's correlations between intrapair differences in dietary intake at 9 years and intrapair differences in subsequent BMIs. This analysis helped us identify the dietary constituents that, when consumed in larger (or smaller) quantities by one twin compared to his/her co-twin, may have led to larger BMI differences between twin pairs (i.e., independent of shared genetic and other environmental influences).

Analyses of intrapair differences indicated that EI at 9 years was not associated with BMI at subsequent ages. For all twins, the proportion of carbohydrates was negatively, and the proportion of protein was positively, associated with BMI change between ages 9 and 14 years. These relationships were driven by a negative association between fruit juice and BMI change, and positive associations between meat and alternatives, and milk and alternatives and BMI change (Table 2). We also observed sex-specific patterns in the associations between macronutrient intake and BMI (Table 3). Carbohydrates were negatively associated, and fats were positively associated, with boys' BMI at all subsequent ages, whereas macronutrient differences were not 
TABLE 1

Descriptive Daily Dietary Intake for MZ Twins at 9 Years ${ }^{\mathrm{a}}$, BMI by Age, and BMI Change $(\Delta)$ Between Ages (Mean and SD), All Twins and By Sex

\begin{tabular}{|c|c|c|c|c|c|c|c|c|c|}
\hline \multirow[b]{2}{*}{ Dietary intake at 9 years and BMI from 9 to 14 years } & \multicolumn{3}{|c|}{ All MZ twin individuals } & \multicolumn{3}{|c|}{ MZ boys } & \multicolumn{3}{|c|}{ MZ girls } \\
\hline & $n$ & Mean & $S D$ & $n$ & Mean & $S D$ & $n$ & Mean & $S D$ \\
\hline Energy (kcal) & 304 & 1814.37 & 393.20 & 138 & $1957.55^{*}$ & 379.75 & 166 & $1695.35^{*}$ & 364.38 \\
\hline Carbohydrates (g) & 304 & 250.87 & 58.81 & 138 & $269.84^{*}$ & 58.48 & 166 & $235.10^{*}$ & 54.41 \\
\hline$\%$ of energy & & 55.46 & 6.45 & & 55.22 & 6.07 & & 55.65 & 6.76 \\
\hline Fats $(\mathrm{g})$ & 304 & 64.91 & 19.30 & 138 & $69.89 *$ & 17.84 & 166 & $60.77^{*}$ & 19.53 \\
\hline$\%$ of energy & & 32.08 & 5.92 & & 32.07 & 4.86 & & 32.09 & 6.68 \\
\hline Proteins (g) & 304 & 64.94 & 18.33 & 138 & $70.00 *$ & 18.74 & 166 & $60.72^{*}$ & 16.91 \\
\hline$\%$ of energy & & 14.30 & 2.57 & & 14.28 & 2.57 & & 14.32 & 2.58 \\
\hline Meat and alternatives (kcal) & 304 & 256.63 & 126.90 & 138 & $281.38^{*}$ & 130.66 & 166 & $236.05^{*}$ & 120.24 \\
\hline$\%$ of energy & & 14.15 & 6.16 & & 14.33 & 5.81 & & 14.01 & 6.46 \\
\hline High-fat meat (kcal) & 304 & 138.97 & 106.26 & 138 & 149.42 & 120.42 & 166 & 130.29 & 92.33 \\
\hline$\%$ of energy & & 7.68 & 5.52 & & 7.60 & 5.61 & & 7.75 & 5.46 \\
\hline High-fat processed meat (kcal) & 304 & 58.31 & 63.31 & 138 & 69.42 & 68.69 & 166 & 50.73 & 51.32 \\
\hline$\%$ of energy & & 3.35 & 3.57 & & 3.66 & 3.92 & & 3.09 & 3.24 \\
\hline Grain products (kcal) & 304 & 586.96 & 207.72 & 138 & $627.33^{*}$ & 220.24 & 166 & $553.40^{*}$ & 190.96 \\
\hline$\%$ of energy & & 32.49 & 9.42 & & 32.05 & 9.34 & & 32.85 & 9.49 \\
\hline Whole grain (kcal) & 304 & 86.37 & 105.31 & 138 & $109.18^{*}$ & 122.44 & 166 & $67.41^{*}$ & 84.38 \\
\hline$\%$ of energy & & 4.78 & 5.85 & & $5.69^{*}$ & 6.49 & & 4.03* & 5.16 \\
\hline Non-whole grain (kcal) & 304 & 500.59 & 204.80 & 138 & 518.15 & 217.18 & 166 & 485.99 & 193.46 \\
\hline$\%$ of energy & & 27.71 & 9.87 & & 26.37 & 9.18 & & 28.82 & $10.31^{*}$ \\
\hline Vegetables (kcal) & 304 & 97.58 & 75.13 & 138 & 111.99* & 79.92 & 166 & $85.61^{*}$ & 70.87 \\
\hline$\%$ of energy & & 5.30 & 3.94 & & $5.72^{*}$ & 4.02 & & $4.95^{*}$ & 3.85 \\
\hline Potatoes (kcal) & 304 & 55.59 & 67.07 & 138 & $68.38^{*}$ & 75.02 & 166 & $45.95^{*}$ & 57.77 \\
\hline$\%$ of energy & & 3.00 & 3.65 & & 3.52 & 3.98 & & $2.57^{*}$ & 3.30 \\
\hline Fruit and fruit juice (kcal) & 304 & 155.59 & 116.44 & 138 & 156.62 & 121.03 & 166 & 154.74 & 112.84 \\
\hline$\%$ of energy & & 8.78 & 6.53 & & 8.13 & 6.24 & & 9.31 & 7.73 \\
\hline Fruit juice only (kcal) & 304 & 79.51 & 83.26 & 138 & 78.68 & 81.29 & 166 & 80.19 & 85.10 \\
\hline$\%$ of energy & & 4.45 & 4.55 & & 4.10 & 4.40 & & 4.75 & 4.67 \\
\hline Milk and alternatives (kcal) & 304 & 318.72 & 154.99 & 138 & 332.35 & 149.93 & 166 & 307.39 & 158.63 \\
\hline$\%$ of energy & & 17.60 & 7.62 & & 17.13 & 7.40 & & 17.98 & 7.79 \\
\hline High-fat milk and alternatives (kcal) & 304 & 147.25 & 126.10 & 138 & 150.84 & 124.67 & 166 & 144.27 & 127.57 \\
\hline$\%$ of energy & & 8.17 & 6.72 & & 7.85 & 6.54 & & 8.49 & 6.86 \\
\hline Low-fat milk and alternatives (kcal) & 304 & 171.47 & 125.81 & 138 & 181.52 & 130.75 & 166 & 163.11 & 121.31 \\
\hline$\%$ of energy & & 9.43 & 6.31 & & 9.28 & 6.46 & & 9.54 & 6.19 \\
\hline Milk (kcal) & 304 & 167.04 & 128.42 & 138 & 175.43 & 130.08 & 166 & 160.07 & 127.00 \\
\hline$\%$ of energy & & 9.13 & 6.52 & & 9.06 & 6.69 & & 9.19 & 6.40 \\
\hline High-fat milk (kcal) & 304 & 25.76 & 83.02 & 138 & 26.60 & 79.99 & 166 & 25.06 & 85.70 \\
\hline$\%$ of energy & & 1.45 & 4.65 & & 1.54 & 4.83 & & 1.38 & 4.51 \\
\hline Low-fat milk (kcal) & 304 & 141.29 & 118.45 & 138 & 148.83 & 122.66 & 166 & 135.01 & 114.84 \\
\hline$\%$ of energy & & 7.68 & 5.93 & & 7.52 & 5.95 & & 7.82 & 5.92 \\
\hline Sugary drinks (kcal) & 304 & 74.22 & 81.95 & 138 & 86.98 & 94.59 & 166 & 63.64 & 68.22 \\
\hline$\%$ of energy & & 4.01 & 4.30 & & 4.36 & 4.59 & & 3.72 & 4.04 \\
\hline Fruit drinks (kcal) & 304 & 47.32 & 64.32 & 138 & 49.78 & 69.93 & 166 & 45.27 & 59.39 \\
\hline$\%$ of energy & & 2.58 & 3.49 & & 2.51 & 3.45 & & 2.64 & 3.53 \\
\hline Soft drinks (kcal) & 304 & 21.27 & 42.48 & 138 & $30.18^{*}$ & 52.75 & 166 & $13.87^{*}$ & 29.69 \\
\hline$\%$ of energy & & 1.14 & 2.22 & & 1.51 & 2.63 & & 0.83 & 1.76 \\
\hline Sweets/desserts/snacks (kcal) & 304 & 191.32 & 144.39 & 138 & $219.31^{*}$ & 152.69 & 166 & $168.04^{*}$ & 133.15 \\
\hline$\%$ of energy & & 10.34 & 7.11 & & 11.08 & 7.46 & & 9.73 & 6.78 \\
\hline BMI 9 years & 304 & 16.51 & 2.50 & 140 & $16.73^{*}$ & 2.15 & 164 & $16.31 *$ & 2.76 \\
\hline BMI 12 years & 294 & 19.21 & 3.62 & 130 & 18.94 & 3.13 & 164 & 19.43 & 3.96 \\
\hline BMI 13 years & 278 & 19.94 & 3.70 & 129 & $19.49^{*}$ & 3.50 & 149 & $20.33^{*}$ & 3.84 \\
\hline BMI 14 years & 276 & 20.68 & 3.63 & 130 & 20.44 & 3.32 & 146 & 20.89 & 3.88 \\
\hline$\Delta \mathrm{BMI} 9$ to 12 years & 238 & 2.46 & 1.79 & 103 & 2.25 & 1.77 & 135 & 2.62 & 1.80 \\
\hline$\Delta \mathrm{BMI} 12$ to 13 years & 243 & 0.85 & 1.47 & 108 & $0.64 *$ & 1.47 & 135 & $1.03^{*}$ & 1.44 \\
\hline$\Delta \mathrm{BMI} 13$ to 14 years & 237 & 0.85 & 1.43 & 114 & 0.88 & 1.48 & 123 & 0.82 & 1.38 \\
\hline$\Delta \mathrm{BMI} 9$ to 14 years & 213 & 4.06 & 2.34 & 92 & $3.75^{*}$ & 2.51 & 121 & $4.29 *$ & 2.19 \\
\hline
\end{tabular}

Note: $\mathrm{BMI}=$ body mass index; $\mathrm{MZ}=$ monozygotic; $\mathrm{SD}=$ standard deviation.

${ }^{a}$ Mean age at 9 years: Boys 9.01 (SD 0.55); Girls 8.93 (SD 0.57).

$* p<.05$ for the difference between boys and girls.

associated with girls' BMI at subsequent ages. For the food groups, analysis of BMI change from 9 to 14 years indicated a positive predictive association for milk and alternatives and a negative one for fruit and fruit juice in boys, and a positive influence for low-fat milk and alternatives (mainly milk and low-fat milk) in girls.

\section{Dietary Intake in Heavier and Leaner Co-Twins}

We first identified BMI-discordant MZ twin pairs at different ages. Table 4 presents the BMI (means and SD) for leaner and heavier discordant (difference of $2 \mathrm{BMI}$ units or more) and concordant (difference of less than 2 BMI units) MZ twins for all twins and by sex, at different ages. Two 
TABLE 2

Spearman's Correlations Between Intrapair Differences $(\Delta)$ in Dietary Intake at 9 Years and Intrapair Differences $(\Delta)$ in BMI at 9, $12,13,14$ Years, and in BMI Change From 9 to 14 years, for All MZ Twin Pairs

\begin{tabular}{|c|c|c|c|c|c|}
\hline \multirow[b]{2}{*}{$\Delta$ Dietary intake at 9 years } & \multicolumn{5}{|c|}{ All } \\
\hline & $\begin{array}{l}\Delta \text { BMI } 9 \text { years } \\
n=151 \text { pairs }\end{array}$ & $\begin{array}{l}\Delta \text { BMI } 12 \text { years } \\
n=119 \text { pairs }\end{array}$ & $\begin{array}{l}\Delta \text { BMI } 13 \text { years } \\
n=113 \text { pairs }\end{array}$ & $\begin{array}{l}\Delta \text { BMI } 14 \text { years } \\
n=106 \text { pairs }\end{array}$ & $\begin{array}{l}\Delta \mathrm{BMI} \text { change } 9-14 \text { years } \\
n=105 \text { pairs }\end{array}$ \\
\hline$\Delta$ Energy (kcal) & 0.13 & 0.07 & 0.10 & 0.07 & 0.00 \\
\hline$\Delta$ Carbohydrates $(\mathrm{g})$ & 0.05 & -0.10 & -0.01 & -0.09 & -0.10 \\
\hline \%of energy & $-0.15^{*}$ & $-0.30^{* *}$ & $-0.17^{*}$ & $-0.32^{* *}$ & $-0.23^{* *}$ \\
\hline$\Delta$ Fats $(\mathrm{g})$ & $0.21^{* *}$ & $0.25^{* *}$ & $0.19^{* *}$ & $0.25^{* *}$ & 0.07 \\
\hline$\%$ of energy & $0.20^{* *}$ & $0.29 * *$ & $0.20^{* *}$ & $0.30^{* *}$ & 0.07 \\
\hline$\Delta$ Proteins $(\mathrm{g})$ & 0.11 & 0.13 & 0.12 & 0.12 & 0.15 \\
\hline$\%$ of energy & 0.03 & 0.14 & 0.08 & 0.13 & $0.23^{* *}$ \\
\hline$\Delta$ Meat and alternatives (kcal) & 0.02 & $0.18^{*}$ & 0.09 & 0.10 & 0.13 \\
\hline$\%$ of energy & -0.04 & 0.13 & 0.04 & 0.09 & $0.17^{*}$ \\
\hline$\Delta$ High-fat meat (kcal) & 0.10 & $0.24^{* *}$ & $0.22^{* *}$ & $0.19 *$ & 0.06 \\
\hline$\%$ of energy & 0.05 & $0.18^{*}$ & $0.16^{*}$ & $0.17^{*}$ & 0.07 \\
\hline$\Delta$ High-fat processed meat (kcal) & 0.02 & 0.04 & 0.04 & 0.15 & 0.10 \\
\hline$\%$ of energy & -0.00 & 0.02 & 0.01 & 0.14 & 0.08 \\
\hline$\Delta$ Grain products (kcal) & $0.27^{* *}$ & 0.04 & 0.04 & -0.03 & -0.16 \\
\hline$\%$ of energy & $0.20^{* *}$ & 0.03 & 0.03 & -0.01 & -0.12 \\
\hline$\Delta$ Whole grain (kcal) & 0.04 & 0.04 & 0.08 & 0.06 & 0.04 \\
\hline$\%$ of energy & 0.00 & 0.03 & 0.06 & 0.04 & 0.00 \\
\hline$\Delta$ Non-whole grain (kcal) & $0.26^{* *}$ & 0.04 & 0.00 & -0.03 & -0.16 \\
\hline$\%$ of energy & $0.20^{* *}$ & 0.06 & 0.02 & -0.03 & -0.13 \\
\hline$\Delta$ Vegetables (kcal) & -0.03 & 0.08 & 0.11 & 0.07 & 0.02 \\
\hline$\%$ of energy & -0.06 & 0.06 & 0.09 & 0.03 & 0.01 \\
\hline$\Delta$ Potatoes (kcal) & -0.03 & 0.05 & 0.10 & 0.05 & -0.00 \\
\hline$\%$ of energy & -0.04 & 0.05 & 0.08 & 0.04 & -0.00 \\
\hline$\Delta$ Fruit and fruit juice (kcal) & -0.09 & $-0.17^{*}$ & $-0.17^{*}$ & -0.16 & -0.13 \\
\hline$\%$ of energy & $-0.14^{*}$ & $-0.22^{* *}$ & $-0.18^{*}$ & $-0.17^{*}$ & -0.14 \\
\hline$\Delta$ Fruit juice only (kcal) & 0.04 & -0.14 & $-0.17^{*}$ & -0.10 & $-0.21^{* *}$ \\
\hline$\%$ of energy & -0.01 & -0.15 & -0.14 & -0.14 & $-0.21^{* *}$ \\
\hline$\Delta$ Milk and alternatives (kcal) & 0.06 & 0.10 & 0.07 & 0.09 & 0.16 \\
\hline$\%$ of energy & -0.02 & 0.08 & 0.08 & 0.08 & $0.21^{* *}$ \\
\hline$\Delta$ High-fat milk and alternatives (kcal) & 0.13 & 0.08 & 0.01 & 0.04 & 0.01 \\
\hline$\%$ of energy & 0.12 & 0.06 & 0.02 & 0.04 & 0.01 \\
\hline$\Delta$ Low-fat milk and alternatives (kcal) & -0.09 & 0.03 & 0.11 & 0.10 & $0.22^{* *}$ \\
\hline$\%$ f energy & -0.13 & 0.05 & 0.12 & 0.12 & $0.26^{* *}$ \\
\hline$\Delta$ Milk (kcal) & 0.02 & 0.13 & 0.10 & 0.12 & $0.16^{*}$ \\
\hline$\%$ of energy & -0.06 & 0.10 & 0.09 & 0.14 & $0.23^{* *}$ \\
\hline$\Delta$ High-fat milk (kcal) & $0.18^{* *}$ & 0.06 & -0.08 & 0.06 & -0.04 \\
\hline$\%$ of energy & $0.17^{* *}$ & 0.05 & -0.08 & 0.06 & -0.03 \\
\hline$\Delta$ Low-fat milk (kcal) & -0.04 & 0.10 & 0.12 & 0.12 & $0.18^{*}$ \\
\hline$\%$ of energy & -0.08 & 0.10 & 0.11 & 0.14 & $0.24^{* *}$ \\
\hline$\Delta$ Sugary drinks (kcal) & 0.01 & -0.02 & -0.00 & -0.06 & -0.09 \\
\hline$\%$ of energy & -0.00 & -0.04 & -0.03 & -0.05 & -0.08 \\
\hline$\Delta$ Fruit drinks (kcal) & -0.03 & -0.11 & -0.12 & -0.13 & -0.12 \\
\hline$\%$ of energy & -0.05 & -0.10 & -0.12 & -0.13 & -0.11 \\
\hline$\Delta$ Soft drinks (kcal) & 0.00 & 0.08 & 0.13 & 0.04 & 0.06 \\
\hline$\%$ of energy & -0.03 & 0.07 & 0.08 & 0.01 & 0.07 \\
\hline$\Delta$ Sweets/desserts/snacks (kcal) & -0.01 & -0.08 & -0.05 & -0.01 & -0.09 \\
\hline$\%$ of energy & -0.06 & -0.12 & -0.11 & -0.02 & -0.07 \\
\hline
\end{tabular}

Note: $\mathrm{BMI}=$ body mass index; $\mathrm{MZ}=$ monozygotic

$* 0.05 \leq p<.10$.

** $p<.05$.

tendencies emerge from these data. First, discordant twins were heavier than concordant twins. Second, MZ discordant twin girls were heavier than same-age MZ discordant twin boys. The BMIs of the heavier co-twins of the concordant pairs were higher than in the heavier co-twins of the concordant pairs for both sexes and for all ages (except for girls at age 14). Moreover, the BMIs of the leaner co-twins of the discordant pairs were higher than in the heavier co-twins of the concordant pairs for all MZ twins and for girls at ages 9 and 13. It is worth noting that there was no association between BMI discordance at adolescence and birth-weight discordance (data not shown).
The analyses that follow identify dietary constituents that could have contributed to higher weights among some MZ twins compared to their co-twins. We calculated the differences in dietary intake at age 9 between the heavier and leaner discordant twins from each MZ pair for BMI at different ages (see Supplementary Tables: Table S1 for all MZ twins; Table S2 for MZ boys; Table S3 for MZ girls). Table 5 presents dietary intake (at 9 years) for the leaner and heavier MZ discordant and concordant twins and for twin pairs that had been discordant at least once, compared to twin pairs that were always concordant at $9,12,13$, and/or 14 years. Only statistically significant associations are presented. 


\section{TABLE 3}

Spearman's Correlations Between Intrapair Differences $(\Delta)$ in Dietary Intake at 9 years and Intrapair Differences $(\Delta)$ in BMI at 9, 12, 13, 14 Years, and in BMI Change From 9 to 14 years, for MZ Twin Pairs, By Sex

\begin{tabular}{|c|c|c|c|c|c|c|c|c|c|c|}
\hline \multirow[b]{2}{*}{$\begin{array}{l}\text { Dietary intake } \\
\text { at } 9 \text { years }\end{array}$} & \multicolumn{5}{|c|}{ MZ Boys } & \multicolumn{5}{|c|}{ MZ Girls } \\
\hline & $\begin{array}{l}\Delta \text { BMI } \\
9 \text { years } \\
n=69 \text { pairs }\end{array}$ & $\begin{array}{l}\Delta \text { BMl } \\
12 \text { years } \\
n=51 \text { pairs }\end{array}$ & $\begin{array}{l}\Delta \text { BMI } \\
13 \text { years } \\
n=48 \text { pairs }\end{array}$ & $\begin{array}{l}\Delta \text { BMI } \\
14 \text { years } \\
n=46 \text { pairs }\end{array}$ & $\begin{array}{l}\Delta \text { BMl change } \\
9-14 \text { years } \\
n=46 \text { pairs }\end{array}$ & $\begin{array}{l}\Delta \text { BMl } \\
9 \text { years } \\
n=82 \text { pairs }\end{array}$ & $\begin{array}{l}\Delta \text { BMI } \\
12 \text { years } \\
n=68 \text { pairs }\end{array}$ & $\begin{array}{l}\Delta \text { BMI } \\
13 \text { years } \\
n=65 \text { pairs }\end{array}$ & $\begin{array}{l}\Delta \mathrm{BMI} \\
14 \text { years } \\
n=60 \text { pairs }\end{array}$ & $\begin{array}{l}\Delta \mathrm{BMI} \text { change } \\
9-14 \text { years } \\
n=59 \text { pairs } \\
\end{array}$ \\
\hline Energy (kcal) & $0.22^{*}$ & 0.09 & 0.21 & 0.07 & -0.01 & 0.05 & 0.08 & 0.05 & 0.11 & 0.03 \\
\hline Carbohydrates (g) & 0.09 & $-0.23^{*}$ & -0.04 & -0.20 & -0.17 & 0.01 & -0.02 & 0.02 & -0.00 & -0.01 \\
\hline \%of energy & $-0.22^{*}$ & $-0.53^{* *}$ & $-0.41 * *$ & $-0.43^{* *}$ & $-0.38^{* *}$ & -0.09 & -0.14 & 0.03 & -0.19 & -0.04 \\
\hline Fats $(g)$ & $0.26^{* *}$ & $0.36^{* *}$ & $0.36 * *$ & $0.34^{* *}$ & $0.28^{*}$ & 0.17 & 0.16 & 0.08 & 0.20 & -0.09 \\
\hline$\%$ of energy & $0.23^{*}$ & $0.51^{* *}$ & $0.42^{* *}$ & $0.41^{* *}$ & $0.32^{* *}$ & 0.18 & 0.14 & 0.06 & 0.21 & -0.15 \\
\hline Proteins (g) & $0.27^{* *}$ & 0.16 & $0.28^{*}$ & 0.19 & 0.15 & -0.05 & 0.11 & 0.01 & 0.06 & 0.15 \\
\hline$\%$ of energy & 0.19 & 0.18 & 0.15 & 0.24 & 0.21 & -0.11 & 0.11 & 0.02 & 0.00 & 0.19 \\
\hline Meat and alternatives(kcal) & $0.20^{*}$ & $0.33^{* *}$ & $0.30^{* *}$ & 0.21 & 0.14 & -0.18 & 0.06 & -0.10 & -0.02 & 0.10 \\
\hline$\%$ of energy & 0.15 & $0.30^{* *}$ & $0.27^{*}$ & 0.20 & 0.15 & $-0.23^{* *}$ & 0.02 & -0.16 & 0.01 & 0.19 \\
\hline High-fat meat (kcal) & $0.24 * *$ & $0.32^{* *}$ & $0.44^{* *}$ & 0.20 & 0.04 & -0.05 & $0.22^{*}$ & 0.03 & 0.21 & 0.10 \\
\hline$\%$ of energy & 0.15 & $0.26^{*}$ & $0.37^{* *}$ & 0.15 & 0.03 & -0.08 & 0.15 & -0.03 & $0.23^{*} \dagger$ & 0.16 \\
\hline High-fat processed meat (kcal) & 0.04 & 0.21 & 0.23 & $0.25^{*} \dagger$ & 0.16 & 0.01 & -0.08 & -0.15 & 0.02 & 0.03 \\
\hline$\%$ of energy & 0.00 & 0.18 & 0.17 & 0.21 & 0.12 & 0.01 & -0.08 & -0.17 & 0.05 & 0.03 \\
\hline Grain products (kcal) & 0.18 & -0.20 & -0.21 & -0.15 & -0.21 & $0.32^{* *}$ & $0.27^{* *}$ & $0.28^{* *} \dagger$ & 0.02 & -0.12 \\
\hline$\%$ of energy & 0.07 & $-0.24^{*} \dagger$ & $-0.28^{*} \dagger$ & -0.12 & -0.13 & $0.29^{* *}$ & $0.26 * *$ & $0.31 * * \dagger$ & 0.06 & -0.05 \\
\hline Whole grain (kcal) & 0.04 & -0.05 & -0.08 & 0.05 & -0.03 & 0.03 & 0.13 & 0.20 & 0.04 & 0.07 \\
\hline$\%$ of energy & -0.02 & -0.06 & -0.08 & 0.05 & -0.02 & 0.04 & 0.13 & 0.15 & 0.01 & -0.00 \\
\hline Non-whole grain (kcal) & 0.20 & -0.14 & -0.17 & -0.12 & -0.16 & $0.31^{* *}$ & $0.21^{*}$ & 0.18 & 0.03 & -0.14 \\
\hline$\%$ of energy & 0.10 & -0.14 & -0.20 & -0.12 & -0.13 & $0.28^{* *}$ & $0.23^{*}$ & $0.23^{*}$ & 0.05 & -0.06 \\
\hline Vegetables (kcal) & -0.01 & 0.16 & 0.08 & 0.18 & 0.14 & -0.06 & -0.01 & 0.17 & 0.00 & -0.05 \\
\hline$\%$ of energy & -0.04 & 0.16 & 0.04 & 0.15 & 0.11 & -0.05 & -0.03 & 0.14 & -0.04 & -0.08 \\
\hline Potatoes (kcal) & -0.03 & 0.15 & 0.11 & 0.15 & 0.08 & -0.03 & -0.05 & 0.14 & -0.02 & -0.06 \\
\hline$\%$ of energy & -0.05 & 0.17 & 0.09 & 0.13 & 0.08 & -0.05 & -0.06 & 0.13 & -0.04 & -0.06 \\
\hline Fruit and fruit juice (kcal) & -0.05 & -0.19 & $-0.37^{* *}$ & $-0.34^{* *}$ & $-0.35^{* *}$ & -0.14 & -0.18 & -0.01 & -0.01 & 0.03 \\
\hline$\%$ of energy & -0.10 & -0.17 & $-0.36^{* *}$ & $-0.31^{* *}$ & $-0.31^{* *}$ & $-0.18^{*} \dagger$ & $-0.25^{* *}$ & -0.05 & -0.05 & 0.00 \\
\hline Fruit juice only (kcal) & -0.03 & -0.13 & $-0.28^{*}$ & -0.24 & $-0.25^{*}$ & 0.10 & -0.16 & -0.11 & 0.03 & -0.18 \\
\hline$\%$ of energy & -0.09 & -0.17 & -0.24 & -0.24 & -0.23 & 0.07 & -0.13 & -0.07 & -0.00 & -0.17 \\
\hline Milk and alternatives (kcal) & 0.13 & 0.06 & 0.18 & 0.18 & 0.20 & -0.04 & 0.14 & -0.02 & 0.00 & 0.12 \\
\hline$\%$ of energy & 0.06 & 0.02 & 0.20 & 0.23 & $0.27^{*}$ & -0.08 & 0.14 & -0.01 & -0.05 & 0.13 \\
\hline High-fat milk and alt. (kcal) & 0.11 & 0.13 & 0.18 & 0.11 & 0.17 & 0.15 & 0.05 & -0.16 & -0.08 & -0.16 \\
\hline$\%$ of energy & 0.10 & 0.12 & 0.19 & 0.15 & 0.18 & 0.13 & 0.02 & -0.13 & -0.09 & -0.15 \\
\hline Low-fat milk and alternatives (kcal) & 0.09 & -0.05 & 0.16 & 0.16 & 0.09 & $-0.25^{* *}$ & 0.10 & 0.12 & 0.09 & $0.32^{* *}$ \\
\hline$\%$ f energy & 0.01 & -0.05 & 0.12 & 0.18 & 0.17 & $-0.28^{* *}$ & 0.12 & 0.16 & 0.12 & $0.37^{* *}$ \\
\hline Milk (kcal) & 0.18 & 0.02 & 0.11 & 0.05 & -0.06 & -0.15 & $0.20^{*}$ & 0.12 & 0.19 & $0.37^{* *}$ \\
\hline$\%$ of energy & 0.08 & -0.01 & 0.09 & 0.10 & 0.01 & $-0.19^{*}$ & 0.19 & 0.14 & $0.24^{*}$ & $0.45^{* *}$ \\
\hline High-fat milk (kcal) & $0.32^{* *}$ & 0.21 & 0.05 & 0.09 & -0.13 & 0.05 & -0.06 & -0.17 & 0.07 & 0.05 \\
\hline$\%$ of energy & $0.31^{* *}$ & 0.21 & 0.05 & 0.10 & -0.12 & 0.05 & -0.07 & -0.18 & 0.06 & 0.06 \\
\hline Low-fat milk (kcal) & 0.08 & -0.05 & 0.11 & 0.06 & -0.02 & -0.15 & $0.24^{*}$ & 0.16 & 0.20 & $0.35^{* *}$ \\
\hline$\%$ of energy & 0.02 & -0.08 & 0.05 & 0.07 & 0.02 & -0.18 & $0.25^{* *}$ & 0.20 & $0.26 * *$ & $0.42^{* *}$ \\
\hline Sugary drinks (kcal) & -0.06 & -0.01 & 0.12 & -0.14 & -0.17 & 0.09 & -0.07 & -0.14 & 0.01 & -0.02 \\
\hline$\%$ of energy & -0.08 & 0.01 & 0.10 & -0.12 & -0.16 & 0.08 & -0.12 & -0.17 & -0.00 & -0.04 \\
\hline Fruit drinks (kcal) & -0.07 & -0.08 & 0.07 & -0.12 & -0.13 & 0.00 & -0.15 & $-0.28^{* *} \dagger$ & -0.16 & -0.09 \\
\hline$\%$ of energy & -0.08 & -0.04 & 0.07 & -0.11 & -0.12 & -0.02 & -0.17 & $-0.26 * * \dagger$ & -0.18 & -0.11 \\
\hline Soft drinks (kcal) & -0.09 & 0.04 & 0.08 & 0.00 & 0.01 & 0.13 & 0.10 & 0.15 & 0.14 & 0.14 \\
\hline$\%$ of energy & -0.12 & 0.03 & 0.02 & -0.01 & 0.01 & 0.09 & 0.07 & 0.11 & 0.08 & 0.15 \\
\hline Sweets/desserts/snacks (kcal) & -0.05 & 0.04 & 0.14 & 0.01 & 0.02 & 0.00 & $-0.21 * \dagger$ & $-0.25^{* *} \dagger$ & -0.04 & $-0.22^{*} \dagger$ \\
\hline$\%$ of energy & 0.11 & 0.02 & 0.10 & -0.03 & 0.01 & -0.02 & $-0.24^{* * \dagger}$ & $-0.30^{* *}$ & -0.01 & -0.18 \\
\hline
\end{tabular}

Note: $\mathrm{BMI}=$ body mass index; $\mathrm{MZ}=$ monozygotic. $* 0.05 \leq p<.10$.

*** $p<.05$.

†Cases where Spearman's correlation was statistically significant because of the presence of extreme bivariate observations; after removing these observations the correlation was no longer significant. 


\section{TABLE 4}

BMI (Mean and Standard Deviation) for All MZ Twins and for Leaner and Heavier Twins From MZ Discordant and Concordant Twin Pairs, All Twins and By Sex and Age, and Cut-Off Values for Overweight or Obesity

\begin{tabular}{|c|c|c|c|c|c|c|c|c|c|c|c|c|c|c|c|c|}
\hline \multirow[b]{3}{*}{$\begin{array}{l}\text { Age group } \\
\text { by sex }\end{array}$} & \multicolumn{5}{|c|}{$\begin{array}{l}\mathrm{MZ} \text { discordant } \mathrm{BMI} \\
\text { (difference of } 2 \mathrm{BMI} \\
\text { units or more) }\end{array}$} & \multicolumn{5}{|c|}{$\begin{array}{l}\text { MZ concordant BMI } \\
\text { (difference of less } \\
\text { than } 2 \text { BMl units) }\end{array}$} & \multicolumn{4}{|c|}{$\begin{array}{l}p \text { value for the comparison } \\
\text { of } M Z \text { discordant (Disc) } \\
\text { and } M Z \text { concordant (Conc) }\end{array}$} & \multicolumn{2}{|c|}{$\begin{array}{l}\text { Cut-off values } \\
\text { for overweight } \\
\text { or obesity }{ }^{b}\end{array}$} \\
\hline & \multirow[b]{2}{*}{$\begin{array}{l}n \\
\text { pairs }\end{array}$} & \multicolumn{2}{|c|}{ Leaner } & \multicolumn{2}{|c|}{ Heavier } & \multirow[b]{2}{*}{$\begin{array}{l}n \\
\text { pairs }\end{array}$} & \multicolumn{2}{|c|}{ Leaner } & \multicolumn{2}{|c|}{ Heavier } & \multirow[b]{2}{*}{$\begin{array}{l}\text { Leaner Disc vs. } \\
\text { Leaner Conc }\end{array}$} & \multirow[b]{2}{*}{$\begin{array}{l}\text { Heavier Disc vs. } \\
\text { Heavier Conc }\end{array}$} & \multirow[b]{2}{*}{$\begin{array}{l}\text { Heavier Disc vs. } \\
\text { Leaner Conc }\end{array}$} & \multirow[b]{2}{*}{$\begin{array}{l}\text { Leaner Disc vs. } \\
\text { Heavier Conc }\end{array}$} & \multirow[b]{2}{*}{ Overweight } & \multirow[b]{2}{*}{ Obese } \\
\hline & & $\begin{array}{l}\text { Mean } \\
\text { BMl }\end{array}$ & $S D$ & $\begin{array}{l}\text { Mean } \\
\text { BMl }^{\mathrm{a}}\end{array}$ & $S D$ & & $\begin{array}{l}\text { Mean } \\
\text { BMI }\end{array}$ & $S D$ & $\begin{array}{l}\text { Mean } \\
\text { BMl }^{\mathrm{a}}\end{array}$ & $S D$ & & & & & & \\
\hline \multicolumn{17}{|l|}{ All } \\
\hline 9 years & 13 & 17.83 & 2.29 & $20.71^{*}$ & 2.66 & 138 & 15.90 & 2.25 & $16.61^{*}$ & 2.34 & $.003^{*}$ & $<.001^{*}$ & $<.001^{*}$ & $.040^{*}$ & $N / A$ & N/A \\
\hline 12 years & 24 & 19.57 & 3.67 & $23.15^{*}$ & 3.79 & 95 & 18.05 & 2.89 & $18.79^{*}$ & 3.03 & .068 & $<.001^{*}$ & $<.001^{*}$ & .400 & $N / A$ & $\mathrm{~N} / \mathrm{A}$ \\
\hline 13 years & 25 & 22.01 & 4.42 & $25.59^{*}$ & 4.58 & 88 & 18.55 & 2.49 & $19.21^{*}$ & 2.52 & $<.001 *$ & $<.001^{*}$ & $<.001 *$ & $.001 *$ & N/A & $\mathrm{N} / \mathrm{A}$ \\
\hline $\begin{array}{l}14 \text { years } \\
\text { Boys }\end{array}$ & 18 & 21.34 & 4.70 & $25.15^{*}$ & 5.30 & 88 & 19.73 & 2.81 & $20.41^{*}$ & 2.82 & .299 & .001 & $<.001^{*}$ & .617 & N/A & N/A \\
\hline 9 years & 9 & 17.00 & 1.95 & $19.46^{*}$ & 2.12 & 60 & 16.16 & 2.02 & $16.89^{*}$ & 2.04 & .137 & $.004^{*}$ & $.001^{*}$ & .662 & 19.10 & 22.07 \\
\hline 12 years & 12 & 19.14 & 3.01 & $23.25^{*}$ & 3.09 & 39 & 18.03 & 2.58 & $18.69^{*}$ & 2.68 & .194 & $<.001 *$ & $<.001^{*}$ & .673 & 21.22 & 26.02 \\
\hline 13 years & 13 & 20.71 & 3.86 & $24.97 *$ & 4.32 & 35 & 18.43 & 2.71 & $19.10^{*}$ & 2.83 & .051 & $<.001^{*}$ & $<.001 *$ & .178 & 21.91 & 26.84 \\
\hline $\begin{array}{l}14 \text { years } \\
\text { Girls }\end{array}$ & 12 & 20.72 & 3.75 & $24.98^{*}$ & 4.62 & 34 & 19.72 & 2.97 & $20.50^{*}$ & 3.07 & .388 & $.003^{*}$ & $.001^{*}$ & .755 & 22.62 & 27.63 \\
\hline 9 years & 4 & 19.70 & 2.04 & 23.50 & 1.17 & 78 & 15.69 & 2.40 & $16.39^{*}$ & 2.53 & $.007^{*}$ & $.001^{*}$ & $.001^{*}$ & $.014^{*}$ & 19.07 & 22.81 \\
\hline 12 years & 12 & 20.00 & 4.32 & $23.05^{*}$ & 4.52 & 56 & 18.06 & 3.11 & $18.86^{*}$ & 3.27 & .145 & $.001^{*}$ & $<.001^{*}$ & .426 & 21.68 & 26.67 \\
\hline 13 years & 12 & 23.43 & 4.70 & $26.27^{*}$ & 4.95 & 53 & 18.62 & 2.36 & $19.28^{*}$ & 2.33 & $<.001^{*}$ & $<.001$ & $<.001^{*}$ & $.001 *$ & 22.58 & 27.76 \\
\hline 14 years & 6 & 22.59 & 6.43 & $25.48^{*}$ & 6.96 & 54 & 19.73 & 2.73 & $20.36^{*}$ & 2.67 & .666 & .160 & $.047^{*}$ & .777 & 23.34 & 28.57 \\
\hline
\end{tabular}

Note: $\mathrm{BMI}=$ body mass index; $\mathrm{MZ}=$ monozygotic; $\mathrm{SD}=$ standard deviation. ${ }^{\mathrm{a} D i f f e r e n c e ~ b e t w e e n ~ h e a v i e r ~ a n d ~ l e a n e r ~ c o-t w i n s: ~}{ }^{*} p<.05 .{ }^{\mathrm{b}}$ Cole et al. (2000). 
TABLE 5

Comparison of Dietary Intake (at 9 years) Among Leaner and Heavier Twins From Discordant (At Least Once at 9, 12, 13, 14 Years) and Concordant MZ Twin Pairs (Never Discordant at 9, 12, 13, 14 Years), All MZ Twins and By Sex ${ }^{\mathrm{a}}$

\begin{tabular}{|c|c|c|c|c|c|}
\hline \multirow[b]{2}{*}{ Dietary intake at 9 years } & \multicolumn{2}{|c|}{$\begin{array}{l}\text { Discordant MZ } \\
\text { twin pairs }^{\mathrm{b}}\end{array}$} & \multicolumn{2}{|c|}{$\begin{array}{c}\text { Concordant } M Z \\
\text { twin pairs }{ }^{\mathrm{c}}\end{array}$} & \multirow[b]{2}{*}{$p$ value (discordant vs. concordant) } \\
\hline & Leaner & Heavier & Leaner & Heavier & \\
\hline \multicolumn{6}{|l|}{ All ${ }^{d}$} \\
\hline Carbohydrates (\% of energy) & 56.10 & $52.72^{* *}$ & 56.52 & 55.33 & $0.049^{* *}$ \\
\hline Fats $(g)$ & 66.26 & $74.79^{* *}$ & 62.37 & 62.26 & $0.013^{* *}$ \\
\hline (\% of energy) & 31.95 & $34.81^{* *}$ & 31.33 & 31.63 & $0.021^{* *}$ \\
\hline Proteins $(\mathrm{g})$ & 62.77 & $69.49 * *$ & 64.75 & 64.02 & $0.036^{* *}$ \\
\hline (\% of energy) & 13.46 & $14.41^{* *}$ & 14.45 & 14.50 & 0.119 \\
\hline Meat and alternatives (kcal) & 240.69 & $290.19^{* *}$ & 253.24 & 251.88 & $0.038^{* *}$ \\
\hline (\% of energy) & 13.07 & $15.00^{* *}$ & 14.15 & 14.26 & 0.125 \\
\hline High-fat meat (kcal) & 122.02 & $180.19^{* *}$ & 133.57 & 133.18 & $0.015^{* *}$ \\
\hline (\% of energy) & 6.84 & $9.27^{* *}$ & 7.51 & 7.51 & $0.038^{* *}$ \\
\hline Whole grain (kcal) & 69.90 & 87.70 & 95.03 & 84.70 & $0.061^{*}$ \\
\hline Vegetables (kcal) & 111.05 & 108.00 & 84.94 & $99.21^{*}$ & 0.670 \\
\hline (\% of energy) & 5.92 & 5.42 & 4.71 & $5.54^{*}$ & $0.064^{*}$ \\
\hline Potatoes (kcal) & 67.02 & 71.04 & 39.92 & $58.83^{* *}$ & 0.438 \\
\hline (\% of energy) & 3.66 & 3.47 & 2.18 & $3.29 * *$ & $0.019^{* *}$ \\
\hline Fruit and fruit juice (kcal) & 147.10 & $123.52^{*}$ & 173.30 & 156.61 & 0.611 \\
\hline (\% of energy) & 8.16 & $6.45^{* *}$ & 9.93 & 8.99 & 0.122 \\
\hline Sugary drinks (kcal) & 95.83 & 80.03 & 76.62 & $59.16^{*}$ & 0.816 \\
\hline (\% of energy) & 5.12 & 4.09 & 4.16 & $3.31^{*}$ & 0.960 \\
\hline \multicolumn{6}{|l|}{ Boys $^{e}$} \\
\hline Carbohydrates (\% of energy) & 56.60 & $51.31^{* *}$ & 57.07 & $55.00^{*}$ & $0.093^{*}$ \\
\hline Fats $(g)$ & 67.75 & $80.10^{* *}$ & 64.97 & 69.61 & 0.101 \\
\hline (\% of energy) & 31.51 & $35.19^{* *}$ & 30.53 & 31.95 & 0.117 \\
\hline Proteins (g) & 65.08 & $76.30^{* *}$ & 69.17 & 69.95 & $0.056^{*}$ \\
\hline (\% of energy) & 13.35 & $14.93^{* *}$ & 14.39 & 14.36 & $0.046^{* *}$ \\
\hline Meat and alternatives (kcal) & 238.87 & $332.70^{* *}$ & 274.06 & 283.03 & $0.038^{* *}$ \\
\hline (\% energy) & 12.25 & $16.19^{* *}$ & 14.36 & 14.43 & $0.053^{*}$ \\
\hline High-fat meat (kcal) & 121.68 & $205.43^{* *}$ & 134.30 & 146.37 & $0.056^{*}$ \\
\hline (\% of energy) & 6.46 & $9.81^{* *}$ & 7.16 & 7.36 & $0.080^{*}$ \\
\hline High-fat processed meat (kcal) & 64.89 & $98.21^{*}$ & 60.00 & 56.67 & $0.098^{*}$ \\
\hline Grain products (\% of energy) & 31.98 & 31.31 & 33.88 & $30.75^{*}$ & 0.393 \\
\hline Vegetables (kcal) & 122.89 & 123.07 & 86.59 & $123.25^{* *}$ & 0.271 \\
\hline (\% of energy) & 6.42 & 5.82 & 4.50 & $6.42^{* *}$ & 0.221 \\
\hline Potatoes (kcal) & 76.41 & 77.58 & 41.95 & $83.72^{* *}$ & 0.165 \\
\hline (\% of energy) & 4.15 & 3.55 & 2.17 & $4.44^{* *}$ & $0.094^{*}$ \\
\hline Fruit and fruit juice (kcal) & 144.06 & $111.99^{* *}$ & 175.13 & 174.89 & 0.240 \\
\hline (\% energy) & 7.62 & $5.18^{* *}$ & 9.31 & 9.18 & 0.208 \\
\hline Fruit juice only (kcal) & 80.92 & 58.89 & 70.83 & 97.82 & $0.084^{*}$ \\
\hline (\% of energy) & 4.35 & $2.68^{* *}$ & 3.78 & 5.18 & $0.041^{* *}$ \\
\hline Milk and alternatives (\% of energy) & 16.72 & 19.39 & 15.68 & $17.40^{*}$ & 0.927 \\
\hline High-fat milk and alternatives (kcal) & 135.57 & $202.00^{* *}$ & 135.07 & 143.52 & 0.101 \\
\hline (\% of energy) & 7.32 & $10.16^{* *}$ & 7.09 & 7.48 & 0.132 \\
\hline \multicolumn{6}{|l|}{ Girls ${ }^{f}$} \\
\hline Grain products (kcal) & 517.95 & $619.07^{*}$ & 542.52 & 554.04 & 0.106 \\
\hline (\% of energy) & 29.85 & $35.12^{*}$ & 31.94 & $34.01^{*}$ & 0.349 \\
\hline Whole grain (kcal) & 47.48 & $81.37^{* *}$ & 68.24 & 68.60 & $0.068^{*}$ \\
\hline (\% of energy) & 2.99 & $4.40^{*}$ & 4.04 & 4.25 & 0.319 \\
\hline Fruit and fruit juice (kcal) & 151.02 & 138.35 & 172.07 & $144.22 *$ & 0.694 \\
\hline High-fat milk (kcal) & 42.33 & 34.79 & 22.98 & 18.00 & $0.047^{* *}$ \\
\hline$\%$ of energy & 2.38 & $1.69^{* *}$ & 1.35 & 0.96 & $0.046^{* *}$ \\
\hline Sugary drinks (\% of energy) & 5.24 & $3.59^{*}$ & 3.85 & 3.12 & 0.667 \\
\hline Fruit drinks (kcal) & 71.37 & $44.60^{* *}$ & 45.26 & 36.67 & $0.053^{*}$ \\
\hline (\% of energy) & 4.17 & $2.42^{* *}$ & 2.67 & 2.17 & 0.158 \\
\hline Soft drinks (kcal) & 13.30 & 16.20 & 15.23 & 11.91 & $0.089^{*}$ \\
\hline
\end{tabular}

Note: $\mathrm{BMI}=$ body mass index; $\mathrm{MZ}=$ monozygotic

** $p<.05$;

$* 0.05 \leq p<.10$ for the difference between leaner and heavier discordant, or leaner and heavier concordant, twins.

aOnly the dietary constituents that are statistically different are reported.

${ }^{b}$ Discordant: $\geq 2$ BMI units difference between twins in a pair, at least once at 9, 12, 13, and/or 14 years; includes only twins who were always heavier (vs. leaner) if heavier more than once, i.e., excludes pairs in which heavier twins became leaner or vice versa at later ages.

'Concordant: <2 BMI units difference between twins in a pair, at all ages $(9,12,13,14$ years).

${ }^{\mathrm{d}}$ Discordant ( $n=48$ pairs); Concordant ( $n=104$ pairs).

e Discordant ( $n=27$ pairs); Concordant ( $n=42$ pairs).

${ }^{f}$ Discordant ( $n=21$ pairs); Concordant ( $n=62$ pairs). 
These analyses indicate that within discordant pairs, the heavier twins did not consume more energy than their leaner co-twins. Compared to their leaner co-twins, heavier boys from discordant pairs ate fewer carbohydrates, fewer grain and non-whole grain products, and less fruit and fruit juice. They also consumed more fats, proteins, meat and alternatives, high-fat meat, and high-fat milk and alternatives. Compared to their leaner co-twins, heavier girls consumed more grain and whole-grain products and more milk and low-fat milk, but less high-fat milk, fewer sugary drinks, and fewer fruit drinks. Overall, heavier boys from both discordant and concordant pairs consumed fewer carbohydrates as a percentage of total EI than their leaner co-twins. Heavier girls from both discordant and concordant pairs consumed more grain products as a percentage of total EI than their leaner co-twins. For boys, compared to twins from concordant pairs, twins from discordant pairs (irrespective of BMI status) consumed fewer carbohydrates and less fruit juice, and more proteins, meat and alternatives (mainly high-fat meat and processed meat), potatoes, and high-fat milk and alternatives. For girls, twins from discordant pairs consumed less whole grain but more high-fat milk, fruit drinks, and soft drinks.

Table 6 presents an overview of study results. It compares the significant results from analyses of intrapair correlations and $\mathrm{MZ}$ discordant pairs. The results from both types of analysis are quite consistent. They illustrate sex differences mainly for grain products, which are negatively associated with BMI in boys and positively in girls.

\section{Discussion}

The main result of this research is that for both sexes, pre-adolescent diets that include certain foods result in higher BMIs during adolescence. After eliminating potential confounding genetic factors, pre-adolescent boys who ate fewer grain products and fruit and consumed more high-fat meat and milk had higher BMIs during adolescence; preadolescent girls who consumed more grain products and high-fat meat and milk had higher BMIs during adolescence. It is worth noting that EI at 9 years is not related to BMI in subsequent years. Thus, the relationship between dietary intake and BMI may not be driven per se by energy imbalances as much as by the type of food constituents consumed.

The observed differences between MZ boys and MZ girls also merit consideration. In MZ twin boys, fats and proteins were positively, and carbohydrates were negatively, associated with BMI in subsequent years. We did not see any relationship between macronutrient intake and BMI in subsequent years in girls except for fats at 14 years. Higher consumption of high-fat meat and lower consumption of fruit show a constant association with higher BMIs in boys and girls, whereas grain products are negatively associated with BMI in boys but positively in girls. To the best of our knowledge, the sex-specific inverse relation that we observed for grain products and BMI has not been reported in other studies. Indeed, the majority of prospective studies on dietary intake and body weight present sex-adjusted data. Nevertheless, the few prospective studies that did report differences in the relation between dietary intake and body fatness also portrayed male and female adults or children differently. Generally speaking, though, only a few dietary elements were identified (Dubois et al., 2011; Halkjaer et al., 2009; Hasselbalch et al., 2010; Koppes et al., 2009; Summerbell et al., 2009).

At the theoretical level, the strength of this research relies on its focus on two major interrelated determinants of population health: dietary intake and BMI. Both are affected by genetic and environmental influences. Our decision to examine dietary intake just before puberty proved very relevant. It highlighted the effect of dietary intake on BMI in adolescence, a period of rapid growth when individuals genetically at risk for obesity may be more sensitive to obesogenic environments (Burt Solorzano \& McCartney, 2010; Dietz, 1994).

Another strength of our research was its methodology. Because it used data from a population-based twin cohort, we could compare pairs of individuals (i.e., MZ twins) who were genetically identical and matched each other perfectly on age, sex and other shared environmental features. Moreover, our analysis benefited from longitudinal data and repeated measurements, which allowed us to analyze dietary intake and BMI in subsequent years. These features combined to allow us to conduct a natural case-control study that would not be easy to replicate otherwise. More specifically, we used two methods with the MZ twin data: intrapair variability analysis and BMI-discordant twin pair analysis. Overall, results from the intrapair correlation analysis and the discordant twin pair analysis were quite consistent, the only difference being that twins from discordant pairs had higher BMIs. These results suggest that the dietary elements associated with higher BMIs may potentially affect a large proportion of the population, independent of genetics. This link will obviously be even stronger among individuals with genetic predispositions to obesity.

The discordant pair approach is particularly suited to the study of complex diseases such as obesity. One challenge it does present, however, is identifying a sufficient number of discordant pairs. Discordance in MZ twins is, by definition, quite rare; all the more so in childhood when twins live together, share interests, friends and hobbies, and attend the same school. Generally speaking, MZ twin discordance increases with age as adolescent twins develop their own peer networks, acquire partners, and are drawn to particular educational and work environments even while still living in the family home. Later, as adult twins, they no longer live together (Naukkarinen et al., 2014). Nevertheless, as Pietilaïnen et al. (2004) also found, sets of discordant twins can display atypical, albeit significant, body weight 
TABLE 6

Comparison of Significant Results $(p<.05$, and $0.05 \leq p<.10)$ for Intrapair Correlations (Tables 3 and 4) and Discordant MZ Twin Pair Analysis (Tables S1, S2, S3) for Dietary Intake at 9 Years (Total $\mathrm{g}$, kcal or \% of Energy) and Subsequent BMI $(12,13,14,9-14$ Years/At Least Once), All Twins and By Sex

\begin{tabular}{|c|c|c|c|c|c|c|}
\hline & \multicolumn{2}{|l|}{ All } & \multicolumn{2}{|r|}{ Boys } & \multicolumn{2}{|c|}{ Girls } \\
\hline & Intrapair correlations & Discordant MZ & Intrapair correlations & Discordant MZ & Intrapair correlations & Discordant MZ \\
\hline \multicolumn{7}{|l|}{ Energy } \\
\hline Macronutrients & $\begin{array}{l}\text { - Carbohydrates** } \\
+ \text { Fats** } \\
+ \text { Proteins }{ }^{* *}\end{array}$ & $\begin{array}{l}\downarrow \text { Carbohydrates** }^{* *} \\
\uparrow \text { Fats }^{* *} \\
\uparrow \text { Proteins }^{* *}\end{array}$ & $\begin{array}{l}\text { - Carbohydrates** } \\
+ \text { Fats** } \\
+ \text { Proteins* }\end{array}$ & $\begin{array}{l}\downarrow \text { Carbohydrates }^{* *} \\
\uparrow \text { Fats }^{* *} \\
\uparrow \text { Proteins } \\
\end{array}$ & & $\uparrow$ Fats* \\
\hline Meat and alternatives & $\begin{array}{l}+ \text { Meat and alternatives* } \\
+ \text { High-fat meat** }\end{array}$ & $\begin{array}{l}\uparrow \text { Meat and alternatives** } \\
\uparrow \text { High-fat meat* } \\
\uparrow H \text { High-fat processed meat }\end{array}$ & $\begin{array}{l}\text { + Meat and alternatives** } \\
+ \text { High-fat meat** } \\
+ \text { High-fat proc. meat* }\end{array}$ & $\begin{array}{l}\uparrow \text { Meat and alternatives** } \\
\uparrow \text { High-fat meat** } \\
\uparrow \text { High-fat processed meat* }\end{array}$ & + High-fat meat* & \\
\hline Grain products & - Grain products** & $\uparrow$ Whole grain** ${ }^{* *}$ & - Grain products* & $\begin{array}{l}\downarrow \text { Grain products** } \\
\downarrow \text { Non-whole grain* }\end{array}$ & $\begin{array}{l}\text { + Grain products** } \\
\text { + Non-whole grain* }\end{array}$ & $\begin{array}{l}\uparrow \text { Grain products* } \\
\uparrow W h \text { hole grain** }\end{array}$ \\
\hline Vegetables & & $\uparrow$ Potatoes** & & & & $\uparrow$ Potatoes* \\
\hline Fruit and fruit juice & $\begin{array}{l}\text { - Fruit and fruit juice** } \\
\text { - Fruit juice only** }\end{array}$ & $\downarrow$ Fruit and fruit juice ${ }^{* *}$ & $\begin{array}{l}\text { - Fruit and fruit juice** } \\
\text { - Fruit juice only* }\end{array}$ & $\begin{array}{l}\downarrow \text { Fruit and fruit juice }{ }^{* *} \\
\downarrow \text { Fruit juice only* }\end{array}$ & - Fruit and fruit juice** & \\
\hline Milk and alternatives & $\begin{array}{l}\text { + Milk and alternatives** } \\
+ \text { Low-fat milk and alternatives } \\
\text { + Milk** } \\
\text { + Low-fat milk** }\end{array}$ & $\begin{array}{l}\uparrow \text { Milk and alternatives* } \\
\uparrow \text { Low-fat milk and alt..** } \\
\uparrow \text { Low-fat milk* }\end{array}$ & + Milk and alternatives* & $\begin{array}{l}\uparrow \text { Milk and alternatives* } \\
\uparrow H \text { High-fat milk and alternatives** } \\
\uparrow \text { Low-fat milk and alternatives* }\end{array}$ & $\begin{array}{l}\text { + Low-fat milk and alternatives** } \\
+ \text { Milk** } \\
+ \text { Low-fat milk** }\end{array}$ & $\begin{array}{l}\downarrow \text { High-fat milk and alternatives* } \\
\downarrow \text { High-fat milk* } \\
\uparrow \text { Milk** } \\
\uparrow L \text { Low-fat milk* }\end{array}$ \\
\hline Sugary drinks & & $\begin{array}{l}\downarrow \text { Sugary drinks* } \\
\uparrow \text { Soft drinks** }\end{array}$ & & $\uparrow$ Soft drinks* & - Fruit drinks** & $\begin{array}{l}\downarrow \text { Sugary drinks* } \\
\downarrow \text { Fruit drinks** }\end{array}$ \\
\hline Sweets/desserts/snacks & & & & & - Sweet/desserts/ snacks** & \\
\hline
\end{tabular}

Note: $\mathrm{BMI}=$ body mass index; $\mathrm{MZ}=$ monozygotic. + Positive correlation; - Negative correlation. $\uparrow$ Heavier twins consumed more of the dietary element than their co-twins. $\downarrow$ Heavier twins consumed less of the dietary element than their co-twins.

**Associated at $p<.05$ at least once. 
variations. Of 60 pairs of twins who were discordant at least once, we removed 12 pairs from our analysis because twins initially identified as heavier later became leaner. This supports the hypothesis that twins from discordant pairs may have so-called 'variability genes' (Pietilaïnen et al., 2004). Our discordant-pair twins, however, had higher BMIs than twins from concordant pairs, which may indicate that they were genetically more predisposed to obesity than other MZ twins from our cohort. Thus the leaner twin in such discordant pairs represents the 'outlier', as some dietary or other factors are keeping them leaner than their biological propensity. This would be despite an overall higher BMI in such pairs. On the other hand, higher BMI pairs of siblings have a great chance of meeting the definition of discordance, even with the leaner twin being of normal BMI, given that physiological homeostatic mechanisms strongly counter attempts (conscious or otherwise) to maintain a lean BMI or to lose weight. The same mechanism will also seek to reduce intrapair weight differences over time.

As is the case for all studies, our study has limitations. A possible limitation of our study is sample size. We conducted baseline intrapair analyses on 152 twin pairs (at 9 years). At subsequent ages the sample size declined to between 106 and 119 twin pairs, which nevertheless represents a low attrition rate. Although these numbers may appear small compared to other twin studies (Silventoinen et al., 2015), we worked with twins who were all the same age at each measurement, and for whom we had taken multiple measurements over time (Boivin et al., 2013). By contrast, many twin studies analyze samples of adult twin pairs whose ages differ and who do not live together, thus introducing variability due to age and environment into their analyses (Silventoinen et al., 2015). Our analysis of twins from discordant pairs relied on small samples of twin pairs at each age. To analyze a larger sample, we selected twins who had belonged to a discordant pair at least once over these years. We identified 27 pairs of boys and 21 pairs of girls. Because MZ twins from BMI-discordant pairs are rare, discordant $\mathrm{MZ}$ twin pair analyses are often done on small samples (i.e., 10-20 pairs; Lewis et al., 2001; Naukkarinen et al., 2014; Pietilaïnen et al., 2004).

Of course, the number of BMI-discordant twin pairs in a study is determined by the definition used to assess discordance. Naukkarinen et al. (2014), using 3 BMI units in 25-year-old MZ twin pairs, identified 16 obesity-discordant pairs to compare with 11 concordant pairs. We chose to use a 2-BMI unit spread since the mean BMI of our adolescent twins was lower and since discordance at this age is rarer. Nevertheless, for most ages, the significance threshold for mean BMI differences between discordant and concordant twins was 3 units or more. Also, the fact that twins from our discordant twin pairs had higher BMIs than those from concordant twin pairs increased our confidence that the discordance cut-off point was adequate for that age group. Analyses conducted for all twins together expanded the number of twin pairs but did not reveal additional statistical relationships. It would nevertheless be highly advisable to replicate this study after pooling our data with data from comparable twin studies.

Finally, twin studies often raise issues about the generalizability of research results to the general population. The twins from our cohort had features similar to those of larger twin studies (Dubois et al., 2012) and were similar in sociodemographic and parental backgrounds and in parity to singletons of the same age from the same population (Vitaro et al., 2011). In our study, twins' dietary intake was also similar to that of singletons in a provincial survey (Bédard et al., 2008).

In conclusion, our study suggests that messages and interventions directed at obesity prevention could take advantage of sex-specific designs and, eventually, genetic information. Interventions that prove effective for one genetically predisposed subgroup may not be effective for others. Higher fruit consumption seems to be related to lower BMIs in both sexes, whereas higher grain-product consumption appears related to lower BMIs in boys and higher BMIs in girls. These findings, once integrated into intervention strategies, can make tangible contributions to collective efforts to stem or prevent obesity development in adolescence.

\section{Acknowledgments}

We are grateful to the children and parents of the QNTS. The QNTS was supported by grants from the Fonds Québécois de la Recherche sur la Société et la Culture (FQRSC), the Fonds de la Recherche en Santé du Québec (FRSQ), the Social Science and Humanities Research Council of Canada (SSHRC), the National Health Research Development Program (NHRDP), CIHR, and the Sainte-Justine Hospital Research Centre. JK is supported by the Academy of Finland (grant \# 263278). MB is supported by the Canada Research Chair Program.

\section{Supplementary Material}

To view supplementary material for this article, please visit http://dx.doi.org/10.1017/thg.2015.97.

\section{References}

Bédard, B., Dubois, L., Baraldi, R., Plante, N., Courtemanche, R., \& Boucher, M. (2008). L'alimentation des jeunes québécois : Un premier tour de table - Enquête sur la santé dans les collectivités canadiennes (cycle 2.2). Québec, Canada: Institut de la statistique du Québec.

Black, A. E. (2000). Critical evaluation of energy intake using the Goldberg cut-off for energy intake: Basal metabolic rate. A practical guide to its calculation, use and limitations. International Journal of Obesity and Related Metabolic Disorders, 24, 1119-1130. 
Bogl, L. H., Pietiläinen, K. H., Rissanen, A., \& Kaprio, J. (2009). Improving the accuracy of self-reports on diet and physical exercise: The co-twin control method. Twin Research and Human Genetics, 12, 531-540.

Boivin, M., Brendgen, M., Dionne, G., Dubois, L., Pérusse, D., Robaey, P., .. V Vitaro, F. (2013). The Quebec newborn twin study into adolescence: 15 years later. Twin Research and Human Genetics, 16, 64-69.

Burt Solorzano, C. M., \& McCartney, C. R. (2010). Obesity and the pubertal transition in girls and boys. Reproduction, 140, 399-410.

Cole, T. J., Bellizzi, M. C., Flegal, K. M., \& Dietz, W. H. (2000). Establishing a standard definition for child overweight and obesity worldwide: international survey. British Medical Journal, 320, 1240-1243.

Connor Gorber, S., Shields, M., Tremblay, M. S., \& McDowell, I. (2009). Correcting self-reported estimates of obesity: Can we more closely approximate measured values?. In Proceedings of Statistics Canada Symposium 2008 - Data collection: Challenges, achievements and new directions. Ottawa, Canada: Statistics Canada.

Crino, M., Sacks, G., Vandevijvere, S., Swinburn, B., \& Neal, B. (2015). The influence on population weight gain and obesity of the macronutrient composition and energy density of the food supply. Current Obesity Reports, 4, 1-10.

Dietz, W. H. (1994). Critical periods in childhood for the development of obesity. American Journal of Clinical Nutrition, 59, 955-959.

Dubois, L., Carter, M. A., Farmer, A., Girard, M., Burnier, D., Tatone-Tokuda, F., \& Porcherie, M. (2011). Higher intakes of energy and grain products at 4 years of age are associated with being overweight at 6 years of age. Journal of Nutrition, 141, 2024-2029.

Dubois, L., Diasparra, M., Bédard, B., Kaprio, J., FontaineBisson, B., Pérusse, D., ... Boivin, M. (2013). Geneenvironment contributions to energy and macronutrient intakes in 9-year-old children: results from the Quebec newborn twin study. Physiology \& Behavior, 119, 30-37.

Dubois, L., Ohm Kyvik, K., Girard, M., Tatone-Tokuda, F., Pérusse, D., Hjelmborg, J., ... Martin, N. G. (2012). Genetic and environmental contributions to weight, height and BMI from birth through 19 years of age: An international study of over 12,000 twin pairs from three continents. PLoS One, 7, e30153.

ESPGHAN Committee on Nutrition, Agostoni, C., Braegger, C., Decsi, T., Kolacek, S., Koletzko, B., ... van Goudoever, J. (2011). Role of dietary factors on food habits in the development of childhood obesity: A commentary by ESPGHAN committee on nutrition. Journal of Pediatric Gastroenterology and Nutrition, 52, 662-669.

Fogelholm, M., Anderssen, S., Gunnarsdottir, I., \& LahtiKoski, M. (2012). Dietary macronutrients and food consumption as determinants of long-term weight change in adult populations: A systematic literature review. Food \& Nutrition Research, 56, 19103.

Garver, W. S., Newman, S. B., Gonzales-Pacheco, D. M., Castillo, J. J., Jelinek, D., Heidenreich, R. A., \& Orlando, R. A. (2013). The genetics of childhood obesity and inter- action with dietary macronutrients. Genes \& Nutrition, 8, 271-287.

Guh, D. P., Zhang, W., Bansback, N., Amarsi, Z., Birmingham, C. L., \& Anis, A. H. (2009). The incidence of co-morbidities related to obesity and overweight: A systematic review and meta-analysis. BMC Public Health, 9, 88.

Hagg, S., Fall, T., Ploner, A., Magi, R., Fischer, K., Draisma, H. H., ... European Network for Genetic and Genomic Epidemiology Consortium. (2015). Adiposity as a cause of cardiovascular disease: A Mendelian randomization study. International Journal of Epidemiology, 44, 578-586.

Halkjaer, J., Tjonneland, A., Overvad, K., \& Sorensen, T. I. (2009). Dietary predictor of 5-year changes in waist circumference. Journal of the American Dietetic Association, 109, 1356-1366.

Hasselbalch, A. L., Heitmann, B. L., Kyvit, K. O., \& Sorensen, T. I. (2010). Associations between dietary intake and body fat independent of genetic and familial environmental background. International Journal of Obesity, 34, 892898.

Health Canada. (2005). Canadian nutrient file (version 2005). Ottawa, Canada: Nutrition Research Division.

Koppes, L. L., Boon, N., Nooyens, A. C., van Mechelen, W., \& Saris, W. H. (2009). Macronutrient distribution over a period of 23 years in relation to energy intake and body fatness. British Journal of Nutrition, 101, 108-115.

Lewis, D. H., Mayberg, H. S., Fischer, M. E., Goldberg, J., Ashton, S., Graham, M. M., \& Buchwald, D. (2001). Monozygotic twins discordant for chronic fatigue syndrome. Radiology, 219, 766-773.

Monteiro, P. O., \& Victora, C. G. (2005). Rapid growth in infancy and childhood and obesity in later life - A systematic review. Obesity Review, 6, 143-154.

Naukkarinen, J., Heinonen, S., Hakkarainen, A., Lundbom, J., Vuolteenaho, K., Saarinen, L., ... Pietiläinen, K. H. (2014). Characterising metabolically healthy obesity in weightdiscordant monozygotic twins. Diabetologia, 57, 167176.

Naukkarinen, J., Rissanen, A., Kaprio, J., \& Pietiläinen, K. H. (2012). Causes and consequences of obesity: The contribution of recent twin studies. International Journal of Obesity, 36, 1017-1024.

Pallister, T., Spector, T. D., \& Menni, C. (2014). Twin studies advance the understanding of gene-environment interplay in human nutrigenomics. Nutrition Research Reviews, 27, 242-251.

Pietiläinen, K. H., Rissanen, A., Laamanen, M., Lindholm, A. K., Markkula, H., Yki-Järvinen, H., \& Kaprio, J. (2004). Growth patterns in young adult monozygotic twin pairs discordant and concordant for obesity. Twin Research, 7, 421-429.

Silventoinen, K., Jelenkovic, A., Sund, R., Honda, C., Aaltonen, S., Yokoyama, Y., ... Kaprio, J. (2015). The CODAtwins project: The cohort description of Collaborative project of development of anthropometrical measures in twins to study macro-environmental variation in genetic and environmental effects on anthropometrical traits. Twin Research and Human Genetics, 18, 348-360. 
Sorensen, T. I. (2009). Challenges in the study of causation of obesity. Proceedings of the Nutrition Society, 68, 43-54.

Speiser, P. W., Rudolf, M. C., Anhalt, H., Camacho-Hubner, C., Chiarelli, F., Eliakim, A., ... Obesity Consensus Working Group. Childhood obesity. (2005). The Journal of Clinical Endocrinology and Metabolism, 90, 1871-1887.

Summerbell, C. D., Douthwaite, W., Whittaker, V., Ells, L. J., Hillier, F., Smith, S., ... Macdonald, I. (2009). The association between diet and physical activity and subsequent excess weight gain and obesity assessed at 5 years of age or older: A systematic review of the epidemiological evidence. International Journal of Obesity, 33, (Suppl. 3), S1S92.

Swinburn, B. A., Sacks, G., Hall, K. D., McPherson, K., Finegood, D. T., Moodie, M. L., \& Gortmaker, S. L. (2011).
The global obesity pandemic: Shaped by global drivers and local environment. The Lancet, 378, 801-814.

Verdonk, P., \& Klinge, I. (2012). Mainstreaming sex and gender analysis in public health genomics. Gender Medicine, 9, 402410.

Vitaro, F., Brendgen, M., Boivin, M., Cantin, S., Dionne, G., Tremblay, R. E., ... Pérusse, D. (2011). A monozygotic twin difference study of friends' aggression and children's adjustment problems. Child Development, 82, 617-632.

Zemel, B. S., Riley, E. M., \& Stallings, V. A. (1997). Evaluation of methodology for nutritional assessment in children: Anthropometry, body composition, and energy expenditure. Annual Review in Nutrition, 17, 211-235. 